\title{
Reliability Analysis of the Random Access Channel of LTE with Access Class Barring for Smart Grid Monitoring Traffic
}

\author{
Charalampos Kalalas and Jesus Alonso-Zarate \\ Centre Tecnològic de Telecomunicacions de Catalunya (CTTC/CERCA), Barcelona, Spain \\ E-mails: \{ckalalas, jesus.alonso\}@cttc.es
}

\begin{abstract}
The efficient and reliable handling of numerous communicating devices responsible for the monitoring and control of the distribution grid constitutes one of the key challenges for the realization of the smart grid. Cellular technology relying on LTE-based standards has been identified as a promising option to support advanced distribution-grid operations with stringent communication requirements. In this paper, a reliability analysis of wide-area monitoring systems in cellular-enabled distribution grids is presented. The LTE random access channel (RACH) procedure enhanced with an access class barring (ACB) scheme is modeled via a Markov chain taking into account a realistic model of the varying traffic behavior of monitoring devices. Based on the proposed analytical framework, we derive the reliability expression which depends on various $\mathrm{RACH}$ and $\mathrm{ACB}$ parameters and the monitoring traffic characteristics. With the aid of extensive simulations, we validate the accuracy of our analytical model. Finally, a performance evaluation in terms of reliability is carried out under different network and traffic configurations and several insights can be drawn for the reliable support of monitoring traffic.
\end{abstract}

\section{INTRODUCTION}

With the emergence of distributed energy resources, the distribution grid integrates more complex loads and decentralized generation, resulting in a growing need for realtime monitoring and control. One of the key components for efficient distribution-grid operation refers to the underlying communication technology selected to support wide-area monitoring systems. These systems typically involve extensive and reliable information exchange originated from monitoring equipment installed along the distribution grid, e.g., Intelligent Electronic Devices (IEDs).

Cellular technology relying on LTE-based standards has been identified as a promising communication option to enhance the observability and controllability of the distribution grid [1]. However, cellular technology was not initially designed to handle efficiently the near-simultaneous access of numerous IEDs. In particular, the LTE Random Access $\mathrm{CHannel}(\mathrm{RACH})$ procedure, where devices request transmission resources or re-establish a connection to the eNodeB, suffers from congestion as the traffic load and the number of access requests increase [2]. Besides the need for network connectivity for a sheer scale of IEDs, the monitoring traffic characteristics, e.g., sporadic and event-driven messages for real-time situational awareness, are fundamentally different from traditional human-type communication.
In this context, the $3 \mathrm{GPP}$ has already raised the need to revisit the radio access design of future cellular networks in order to provide reliable connectivity for massive machinetype communication [3]. Among the various methods proposed to improve the contention-based RACH operation, the 3GPP adopts the Access Class Barring (ACB) scheme as an additional overload control mechanism to prevent access failures [4]. In case of network overload, the eNodeB broadcasts to the different traffic classes a set of parameters related to ACB, as part of the system information; this includes a barring rate factor and a barring timer for backoff. Each device then performs a Bernoulli trial to determine whether it is barred or not, based on the barring rate value. The ACB scheme and its subsequent amendments, e.g., dynamic ACB [5] for adaptive barring and extended ACB [6] for delay prioritization, rely on backoff periods to disperse the simultaneous access attempts over time and alleviate the congestion.

Several simulation-based studies [5], [7]-[9] and analytical works [10], [11] investigate the performance of the LTE RACH procedure for smart grid traffic. The majority of feasibility studies aim at determining the optimal values of the barring [5], [7], backoff [8] or paging [9] RACH parameters through system-level simulations. In an effort to proactively estimate the anticipated smart grid traffic load (alarm reports, periodic measurements), the authors in [10] propose a mechanism that determines the channel access opportunities for smart grid entities. A similar approach is followed in [6], where the authors perform an analysis of the extended ACB scheme based on difference equations in order to estimate the RACH performance. In both works [6], [10], the 3GPP-based models for uncoordinated and synchronous traffic are adopted. Instead, here we assume a reactive approach in dealing with massive monitoring traffic arrivals, where the network loading state is considered to be continuously monitored by the eNodeB with the overload-detection mechanism proposed in [12]. The authors in [11] analytically assess the signaling limitations of the standard RACH procedure for smart grid monitoring traffic. However, no ACB scheme is considered in the analysis and the traffic modeling approach relies on a simple Poisson process for the aggregated data with periodic reporting frequency.

Contribution: The contribution of this paper is threefold. First, by leveraging tools from Markov chain theory, we introduce a tractable analytical model of the contention-based LTE 
random access mechanism enhanced with an ACB scheme for the connection establishment of a high number of IEDs. In addition, we propose a Markov Modulated Poisson Process (MMPP) traffic model to capture the varying traffic behavior of monitoring IEDs in power distribution grids. Unlike previous related works, our traffic modeling approach accounts for the frequency and duration of a burst traffic generation. Finally, we derive the analytical expression of the achieved reliability based on the developed analytical framework. The accuracy of the proposed analysis is validated with the aid of extensive simulations in ns-3 discrete-event simulator. A performance assessment reveals the impact of the monitoring traffic characteristics and $\mathrm{RACH} / \mathrm{ACB}$ parameters in the achieved reliability.

Organization: The rest of the paper is organized as follows. Section II provides an overview of the channel access procedure followed in LTE standards. In Section III, an analytical model of the contention-based LTE random access mechanism enhanced with an ACB scheme is introduced and a traffic model for monitoring IEDs in the distribution grid is proposed. In Section IV, the analytical expression of reliability is derived as a function of the monitoring traffic characteristics and $\mathrm{RACH} / \mathrm{ACB}$ parameters. Our analysis is validated by means of extensive simulations in Section $\mathrm{V}$ along with a performance assessment in terms of reliability under different network and traffic configurations. Section VI concludes the paper.

\section{OvERVIEW OF THE RANDOM ACCESS MECHANISM IN LTE NETWORKS}

In LTE networks, devices use the physical RACH to perform initial association with the eNodeB, re-establish a failed radio link or request transmission resources when no dedicated scheduling-request resources have been assigned [2]. The $\mathrm{RACH}$ is formed by a periodic reservation of uplink timefrequency resource blocks, the random access slots, for the transmission of access requests. The eNodeB broadcasts the random access slot periodicity which may vary from once in every subframe (1ms) to once every two frames $(20 \mathrm{~ms})$, based on the RACH configuration index ${ }^{1}$ [2]. In this study, we assume that a random access slot is available in every subframe to counteract for the high number of requesting IEDs.

The random access procedure in LTE can be either contention-free or contention-based. In the contention-free mode, the eNodeB allocates dedicated access resources for requests that require high probability of success, e.g., handover and synchronization. This study focuses on the contentionbased random access operation enhanced with an ACB scheme. As illustrated in Fig. 1, each IED draws a uniform random number between 0 and 1 when initiating a connection establishment and compares it with the current barring rate ${ }^{1}$; if the number is lower than the barring rate, the IED is able to attempt an access. Otherwise, the access is barred and the IED performs a random backoff time uniformly selected over a period determined by the barring timer value ${ }^{1}$, before performing a new trial.

\footnotetext{
${ }^{1}$ The values of the RACH configuration index and the ACB parameters are broadcast by the eNodeB as part of the system information [13].
}

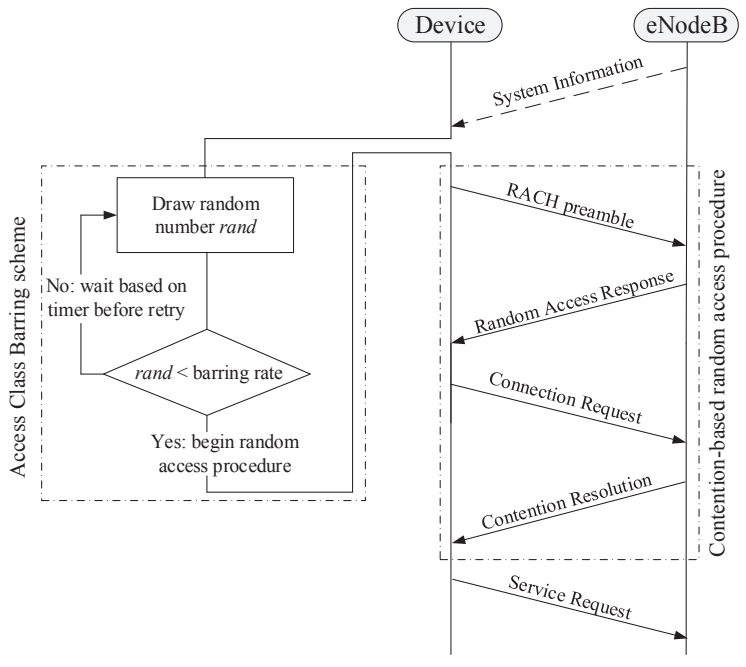

Fig. 1. Contention-based LTE random access procedure enhanced with an ACB scheme [2].

The contention-based random access mechanism involves a four-way message exchange with the eNodeB. In particular, the following consecutive messages are sent:

1) $\mathrm{RACH}$ preamble. According to the $3 \mathrm{GPP}$ physical layer specifications, a preamble constitutes a pseudo-random digital signature that a device randomly selects to transmit over the first available random access slot of the RACH. A subset of preambles is often reserved for contention-free access and their availability for contention-based access is periodically broadcast by the eNodeB.

2) Random Access Response (RAR). In turn, the eNodeB processes the preambles received on a specific random access slot and provides feedback in a RAR message to all the IEDs with preamble transmission on this random access slot. The RAR message includes an identifier of each successfully decoded preamble, timing information for synchronization, a temporary device identifier and an uplink resource grant for devices to transmit a connection request, the next message of the handshake. In case that an IED receives a RAR without the identifier of the preamble it used, it is signaled, via a backoff indicator attached to the RAR, to wait for a random time uniformly selected over a period, until the next preamble transmission attempt. If more than one IEDs attempted access with the same preamble over the same random access slot, they will receive the same RAR message and their contention will be resolved in the next step.

3) Connection Request. Upon receiving the resource grant in RAR, the IED transmits a connection request message to the eNodeB, conveying -among other information- the device identifier and the establishment cause, e.g., scheduling resources request. In case of preamble collision in the previous step, more than one IEDs will transmit in the same uplink resource; the eNodeB then identifies the collision and will not reply with an acknowledgment.

4) Contention Resolution: In response to a successfully received connection request message, the eNodeB transmits a 
contention resolution message as an acknowledgment. The completion of this step renders the random access attempt successful. Otherwise, if the message is not received by an IED within a predefined contention resolution timer, the random access procedure is considered unsuccessful and the IED needs to restart a new random access attempt until the limit for its allowed preamble transmissions is reached.

\section{Analytical Model}

In this section, we propose an analytical model of the contention-based LTE random access mechanism enhanced with an ACB scheme for the efficient handling of a high number of monitoring IEDs. We consider a cellular-enabled power distribution grid where $N$ IEDs reside within a singlecell coverage area and contend in the RACH for network access. In the following, we study the behavior of a single IED by using a two-dimensional Markov chain model.

\section{A. Markov Chain Model}

Let $\alpha(t)$ and $\beta(t)$ be the stochastic processes corresponding to the random access attempt and the backoff stage at time $t$, respectively, experienced by an IED contending for channel access. We assume that the stationary probability $\tau$ that an IED attempts a random access is constant across all random access slots and independent of other IEDs. This is considered as a realistic assumption in the case of a high number of IEDs present in the system. Then, $(\alpha(t), \beta(t))$ constitutes a two-dimensional Markov chain. Let also $M$ be the maximum allowed number of random access attempts, $r_{\text {th }}$ the access class barring rate and $B, W$, the backoff window sizes for barring and random access, respectively. As indicated in Fig. 2, for the finite state space of the Markov chain, the following hold:

- The off state represents the state when the idle IED is expecting a new packet arrival based on the considered unsaturated traffic model. Let $p_{\text {on }}$ be the traffic generation probability related with the specific monitoring application.

- The states $\left(Q_{0}, \ldots, Q_{B-1}\right)$ model the barring states of the ACB scheme. The probability of entering/returning to a barring state equals to the probability $q_{0}$ of the drawn random number being higher than the barring rate $r_{\text {th }}$ of the ACB scheme, normalized by the barring backoff window size, $B$.

- The states $(i, 0), i \in[1, M]$, correspond to the random access states when the IED attempts a preamble transmission. In this work, we assume that an unsuccessful random access attempt may occur only due to preamble collision; thus, the probability of moving from states $(i-1,0)$ to $(i, j)$, for $i \in[2, M]$, equals to the preamble collision probability, $p_{c}$, normalized by the random access backoff window size, $W$. We leave the consideration of a wireless channel error on an unsuccessful preamble transmission and the capture effect of collided connection request messages for future work.

- The states from $(i, 1)$ to $(i, W-1), i \in[2, M]$, represent the backoff states due to an unsuccessful $(i-1,0)$ access

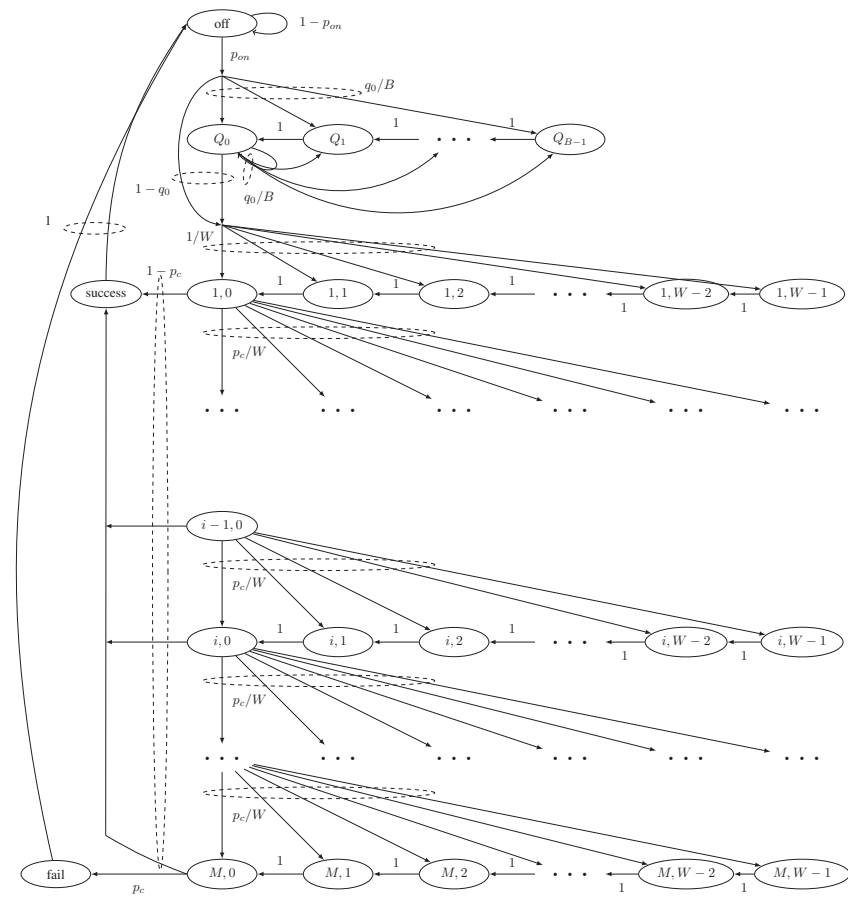

Fig. 2. Markov chain model for the contention-based LTE random access mechanism enhanced with an ACB scheme.

attempt. The IED decreases its backoff index by 1 every $1 \mathrm{~ms}$ and transits from $(i, j)$ to state $(i, j-1)$. A random backoff is also considered upon the initialization of the RACH procedure, i.e., the states $(1, j), j \in[1, W-1]$.

- The success and fail states model the successful and failed random access attempt, respectively. A failed random access attempt occurs when the limit of $M$ allowed preamble transmissions is reached without successful attempt.

- The IED always returns to the off state after the success or fail states.

The state transition probabilities associated with the Markov chain of Fig. 2 can be calculated as follows:

$$
\begin{aligned}
& \operatorname{Pr}(i, j \mid i, j+1)=1,1 \leq i \leq M, 0 \leq j \leq W-2, \\
& \operatorname{Pr}(i, j \mid i-1,0)=\frac{p_{c}}{W}, 2 \leq i \leq M, 0 \leq j \leq W-1, \\
& \operatorname{Pr}\left(Q_{c} \mid Q_{c+1}\right)=1,0 \leq c \leq B-2, \\
& \operatorname{Pr}\left(Q_{c} \mid \text { off }\right)=\frac{q_{0}}{B} p_{\text {on }}, 0 \leq c \leq B-1, \\
& \operatorname{Pr}\left(Q_{c} \mid Q_{0}\right)=\frac{q_{0}}{B}, 0 \leq c \leq B-1, \\
& \operatorname{Pr}(1, j \mid \text { off })=\frac{\left(1-q_{0}\right)}{W} p_{\text {on }}, 0 \leq j \leq W-1, \\
& \operatorname{Pr}\left(1, j \mid Q_{0}\right)=\frac{\left(1-q_{0}\right)}{W}, 0 \leq j \leq W-1, \\
& \operatorname{Pr}(\text { fail } \mid M, 0)=p_{c}, \\
& \operatorname{Pr}(\text { success } \mid i, 0)=1-p_{c}, 1 \leq i \leq M, \\
& \operatorname{Pr}(\text { off } \mid \text { success })=\operatorname{Pr}(\text { off } \mid \text { fail })=1 .
\end{aligned}
$$


Eq. (1a) shows the decrease of the backoff index, which occurs with probability 1 . Eq. (1b) represents the probability of collision in the random access and of selecting a backoff state uniformly in the subsequent random access attempt. Eq. (1c) shows the transition between the barring states which occurs with probability 1 . Eqs. (1d) and (1e) capture the probabilities of entering and returning to the barring states from the off and $Q_{0}$ states, respectively. Eqs. (1f) and (1g) represent the probabilities of moving to state $(1, j)$ from the off and $Q_{0}$ states, respectively. Eqs. (1h) and (1i) capture the probabilities of a failed and successful random access, respectively. Eq. (1j) implies that the IED always returns to off state after a successful or failed random access.

Let $\left(b_{\text {off }}, b_{Q_{c}}, b_{i, j}, b_{\text {success }}, b_{\text {fail }}\right), c \in[0, B-1], i \in[1, M]$, $j \in[0, W-1]$, be the stationary distribution of the Markov chain, where $b_{i, j}=\lim _{t \rightarrow \infty} \operatorname{Pr}(\alpha(t)=i, \beta(t)=j)$. From Eqs. (1a)-(1j), we derive the closed form expression for such distribution chain. In particular, for the stationary probabilities of the barring states we have

$b_{Q_{c}}=\frac{q_{0}}{B} p_{\text {on }} b_{\text {off }}+b_{Q_{c+1}}+\frac{q_{0}}{B} b_{Q_{0}}, 0 \leq c \leq B-2$,

$b_{Q_{B}-1}=\frac{q_{0}}{B} p_{\text {on }} b_{\text {off }}+\frac{q_{0}}{B} b_{Q_{0}}$.

For the random access and backoff states we obtain

$b_{1, j}=b_{1, j+1}+\frac{\left(1-q_{0}\right)}{W}\left(b_{Q_{0}}+p_{o n} b_{\text {off }}\right), 0 \leq j \leq W-2$,

$b_{1, W-1}=\frac{\left(1-q_{0}\right)}{W}\left(b_{Q_{0}}+p_{\text {on }} b_{\text {off }}\right)$,

$b_{i, j}=b_{i, j+1}+b_{i-1,0} \frac{p_{c}}{W}, 2 \leq i \leq M, 0 \leq j \leq W-2$,

$b_{i, W-1}=b_{i-1,0} \frac{p_{c}}{W}, 2 \leq i \leq M$.

Finally, for the success and fail states we have

$b_{\text {success }}=\sum_{i=1}^{M}\left(\left(1-p_{c}\right) b_{i, 0}\right)$,

$b_{\text {fail }}=p_{c} b_{M, 0}$.

Owing to the chain regularities and Eqs. (2)-(9), the state expressions can be rewritten as

$b_{Q_{0}}=\frac{q_{0}}{1-q_{0}} p_{\text {on }} b_{\text {off }}$,

$b_{Q_{c}}=(B-c) \frac{q_{0}}{B}\left(p_{\text {on }} b_{\text {off }}+b_{Q_{0}}\right), 1 \leq c \leq B-1$,

$b_{1, j}=\frac{W-j}{W} b_{1,0}, 1 \leq j \leq W-1$,

$b_{1,0}=\left(1-q_{0}\right)\left(b_{Q_{0}}+p_{\text {on }} b_{\text {off }}\right)$,

$b_{i, j}=\frac{W-j}{W} b_{i, 0}, 2 \leq i \leq M, 1 \leq j \leq W-1$,

$b_{i, 0}=b_{i-1,0} p_{c}=b_{1,0} p_{c}^{i-1}, 2 \leq i \leq M$,

$b_{\text {success }}=p_{\text {on }} b_{\text {off }}\left(1-p_{c}^{M}\right)$,

$b_{\text {fail }}=p_{c}^{M} p_{\text {on }} b_{\text {off }}$.

Note that from Eqs. (10a)-(10h), all states can be expressed as a function of $b_{\text {off. }}$. By applying the expressions for the state

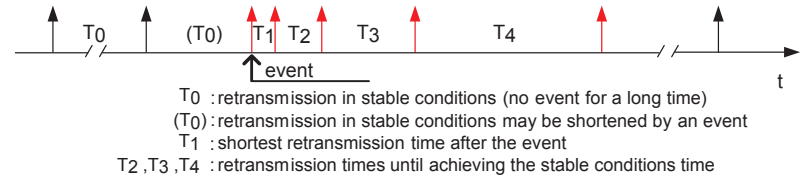

Fig. 3. GOOSE burst traffic pattern [12]. Under stable conditions, GOOSE messages follow a periodic traffic pattern, but are generated in bursts when an event occurs.

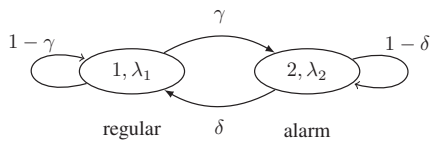

Fig. 4. The MMPP model for each IED traffic generation. A two-state Markov chain describes the transition between the regular and alarm operation of a monitoring IED.

stationary probabilities in the normalization condition for the Markov chain,

$$
1=b_{\text {off }}+\sum_{c=0}^{B-1} b_{Q_{c}}+\sum_{i=1}^{M} \sum_{j=0}^{W-1} b_{i, j}+b_{\text {success }}+b_{\text {fail }},
$$

we obtain the expression for the stationary probability $b_{\text {off }}$,

$$
b_{\text {off }}=\frac{1}{1+p_{\text {on }}\left(1+\frac{B+1}{2} \frac{q_{0}}{1-q_{0}}+\frac{W+1}{2} \frac{p_{c}^{M}-1}{p_{c}-1}\right)},
$$

as a function of the traffic generation probability, $p_{o n}$, the preamble collision probability, $p_{c}$, and the probability of barred access, $q_{0}$.

In the following, we derive the expression for the traffic generation probability, $p_{\text {on }}$, based on a realistic traffic model selected to match the behavior of a monitoring IED.

\section{B. Traffic Model}

The IEDs reside within the substation local area networks and can be seen as controllers that get their input from voltage and current transformers/sensors and provide their output (commands, status data), e.g., to circuit breakers, offering monitoring functionalities in the distribution grid. Under stable operating conditions, each IED periodically reports its application states via identical messages as a heart-beat function; however, once an event (e.g., power failure) occurs or a status change is detected, an IED transits from regular to alarm state while the retransmission period of messages is shortened (burst traffic). Fig. 3 shows the traffic pattern of IEC 61850-8-1 Generic Object Oriented Substation Event (GOOSE) messages that can be used for fast horizontal communication among IEDs to enable fast transmission of substation events [12].

In an effort to capture this varying behavior of IED traffic, we may model each IED traffic generation using the Markov Modulated Poisson Process (MMPP) framework [14]. In particular, each IED is represented by a two-state Markov chain and a corresponding Poisson process. As illustrated in Fig. 4, the first state models the regular IED operation as a Poisson process with rate $\lambda_{1}$ and the second state represents the alarm IED operation where the generation of a traffic burst 
is modeled as a Poisson process with a corresponding rate $\lambda_{2}>\lambda_{1}$. Let $P$ be the state transition matrix that incorporates the transition probabilities between the states of the Markov chain of Fig. 4. Then,

$$
P=\left[\begin{array}{cc}
1-\gamma & \gamma \\
\delta & 1-\delta
\end{array}\right]
$$

where $\gamma$ is associated with the frequency of a burst occurrence and $\delta$ is related with the duration of each burst. Let also $\pi=$ $\left\{\pi_{1}, \pi_{2}\right\}$ be the stationary distribution vector. Then, from the steady-state equations $\pi=\pi P$ and $\pi_{1}+\pi_{2}=1$, we derive the stationary distribution $\pi$ of the chain as

$$
\pi=\left\{\frac{\delta}{\gamma+\delta}, \frac{\gamma}{\gamma+\delta}\right\}
$$

The traffic generation probability for an IED, $p_{\text {on }}$, is defined as the probability to have at least one packet arrival within a subframe of length $1 \mathrm{~ms}$, and is calculated as

$$
p_{\text {on }}=1-e^{-\lambda_{g}} \text {, }
$$

where the $\lambda_{g}=\sum_{i=1}^{2} \lambda_{i} \pi_{i}$ denotes the overall rate of the MMPP [14]. In the following section, we derive an analytical expression for the reliability based on the developed Markov chain model.

\section{RELIABILITY ANALYSiS}

We define reliability, $R$, as the probability of an IED successfully completing a random access attempt when there are $M$ allowed preamble transmission attempts. Based on the proposed Markov chain model developed in the previous section, we derive the expression of reliability as

$$
R=\frac{b_{\text {success }}}{b_{\text {success }}+b_{\text {fail }}},
$$

and by using the expressions for $b_{\text {success }}$ and $b_{\text {fail }}$ from $(10 \mathrm{~g})$ and (10h) respectively, we get

$$
R=1-p_{c}^{M} .
$$

For the calculation of $R$ we first need to determine the probability $\tau$ that an IED attempts for access. Assuming that the preamble transmission holds for $1 \mathrm{~ms}, \tau$ is determined as

$$
\tau=\frac{\sum_{i=1}^{M} b_{i, 0}}{T},
$$

where $T$ denotes the expected state holding time for all states and is equal to

$$
\begin{aligned}
T & =b_{\text {off }}\left(\frac{1}{\lambda_{g}}\right)+\sum_{c=0}^{B-1} b_{Q_{c}}+\sum_{i=1}^{M} \sum_{j=1}^{W-1} b_{i, j} \\
& +\sum_{i=1}^{M} b_{i, 0}\left(p_{c} T_{1}+\left(1-p_{c}\right) T_{2}\right)+b_{\text {success }} T_{s}+b_{\text {fail }} T_{f} .
\end{aligned}
$$

In Eq. (19), the expected holding time for the off state is $1 / \lambda_{g}$ and the expected holding time of each barring and
TABLE I

Simulation PARAMETERS

\begin{tabular}{|lc|}
\hline Parameter & Value \\
\hline Preambles for contention-based access $K$ & 54 \\
Number of monitoring IEDs $N$ & 1000 \\
RACH configuration index & 14 \\
Barring/access backoff window sizes $B, W$ (in ms) & 20 \\
Barring rate $r_{\text {th }}$ & 0.5 \\
Preamble duration (in ms) & 1 \\
Max. allowed preamble transmission attempts M & 10 \\
RAR window size (in ms) & 5 \\
Contention resolution timer (in ms) & 24 \\
Master information block periodicity (in ms) & 40 \\
Arrival rates $\lambda_{1}, \lambda_{2}$ (in attempts/ms) & $\{0.002,0.5\}$ \\
Time durations $T_{1}, T_{2}, T_{s}, T_{f}$ (in ms) & $\{32,16,20,1\}$ \\
Traffic model transition probabilities $\gamma, \delta$ & $\{0.5,0.3\}$ \\
\hline
\end{tabular}

backoff state is $1 \mathrm{~ms}$. The expected holding time of a random access state $(i, 0)$ is obtained as $p_{c} T_{1}+\left(1-p_{c}\right) T_{2}$, where $T_{1}$ and $T_{2}$ correspond to the elapsed times from the first access attempt until the end of the contention resolution timer in case of failure, and until the reception of the contention resolution message in case of success, respectively. Finally, $T_{f}$ denotes the expected time duration of the fail state and $T_{s}$ corresponds to the average holding time of the success state which depends on the adopted resource-scheduling policy of the service request and the message payload of the monitoring IED.

Given that an IED transmits, the preamble collision probability, $p_{c}$, from the perspective of the IED, is defined as the probability that at least one of the $s$ IEDs attempting random channel access (from the remaining $N-1$ IEDs) selects the same preamble. Let $K$ be the number of available preambles for contention-based random access, then $p_{c}$ is given by the conditional probability

$$
p_{c}=\sum_{s=1}^{N-1}\left(\begin{array}{c}
N-1 \\
s
\end{array}\right) \tau^{s}(1-\tau)^{N-1-s}\left(1-\left(1-\frac{1}{K}\right)^{s}\right),
$$

as a function of $\tau$. It can thus be observed from (12)-(15) and (18)-(20), that the reliability expression in (17) depends on the monitoring traffic characteristics and the RACH/ACB parameters. Note that for known $p_{o n}$ and $q_{0}$, the expressions of the probability $\tau$ of attempting a random access in (18) and the preamble collision probability $p_{c}$ in (20) form a system of nonlinear equations that can be solved via an iterative numerical method. Therefore, by plugging the obtained value of $p_{c}$ in (17), the value of $R$ can be calculated.

\section{Model Validation and Performance Evaluation}

In this section, we validate and evaluate our proposed analytical framework in terms of reliability with the aid of extensive simulations with ns-3 discrete-event simulator. The simulation setup relies on the RACH implementation initially developed in [2] and follows the 3GPP specifications [3]. The existing traffic generation and ACB modules have been properly modified/extended when necessary and the MMPP parameters are selected to closely match the traffic behavior 


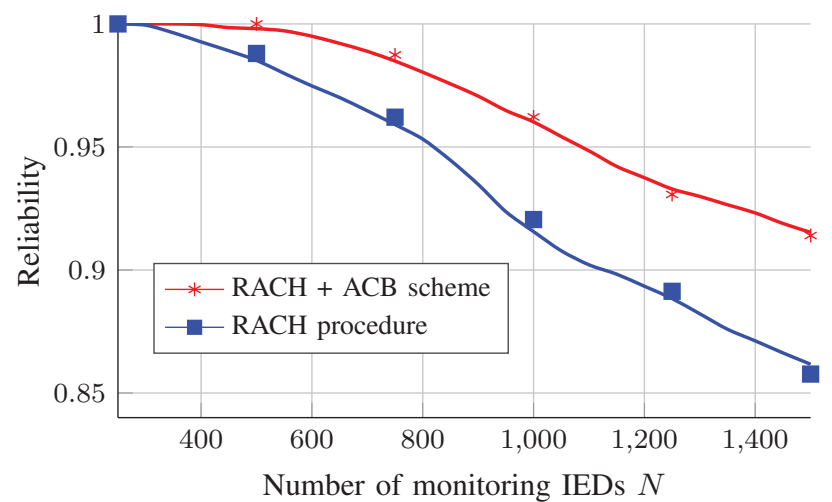

Fig. 5. Reliability achieved per IED for different number of monitoring IEDs present in the system when RACH procedure is enhanced with the ACB scheme and when ACB is not applied.

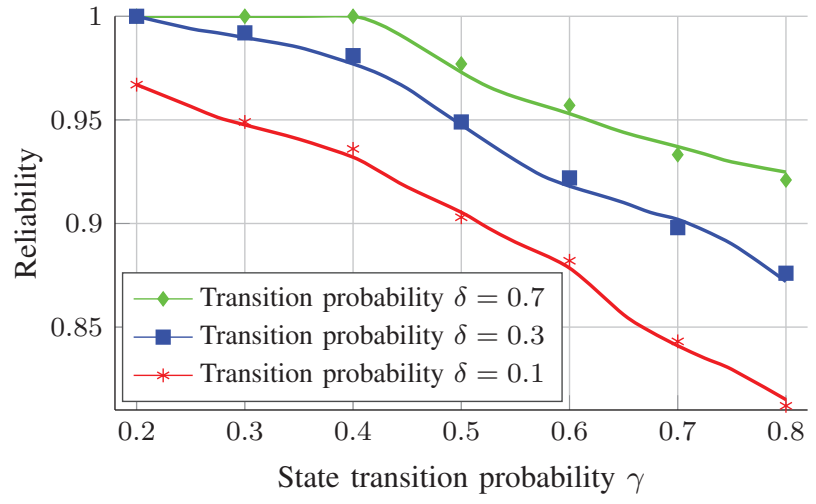

Fig. 6. Reliability achieved per IED as a function of the traffic characteristics $\gamma, \delta$ of the monitoring IEDs when ACB scheme is applied in the RACH.

of IEC 61850 GOOSE messages conveying synchrophasor information [15]. A performance evaluation is conducted and we investigate the effect of the traffic characteristics, the barring rate and the number of available preambles on the achieved reliability. In Figs. 5-8, we illustrate both the analytical results (lines) obtained from the reliability expression in Eq. (17) and the simulation results (marks) of the RACH/ACB implementation. It can be observed that the analytical results accurately match the simulation results which validates our proposed analytical model. Table I summarizes the basic parameters used in our simulations.

Fig. 5 illustrates the reliability achieved per IED for a different number of monitoring IEDs present in the system, when the ACB scheme is activated $\left(q_{0} \neq 0\right)$ and when ACB barring is not applied in the RACH procedure $\left(q_{0}=0\right)$. It can be seen that reliability levels decrease as the number of IEDs increases due to the heavier contention; however, a $\mathrm{RACH}$ procedure enhanced with the ACB scheme achieves higher reliability even in high traffic regime since IED channel access attempts are dispersed over time. Thus, IEDs remain in contention for less time and preamble collision probability is reduced.

Fig. 6 shows the reliability achieved per IED as a function of the traffic characteristics $\gamma, \delta$ of the monitoring IEDs. As defined in Fig. 4, $\gamma$ is associated with the frequency of

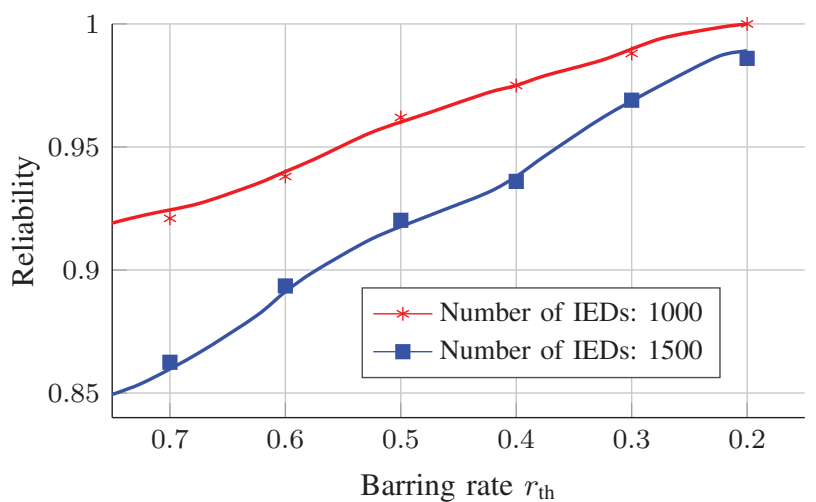

Fig. 7. Reliability achieved per IED as a function of the barring rate $r_{\text {th }}$ of the ACB scheme for a different number of IEDs.

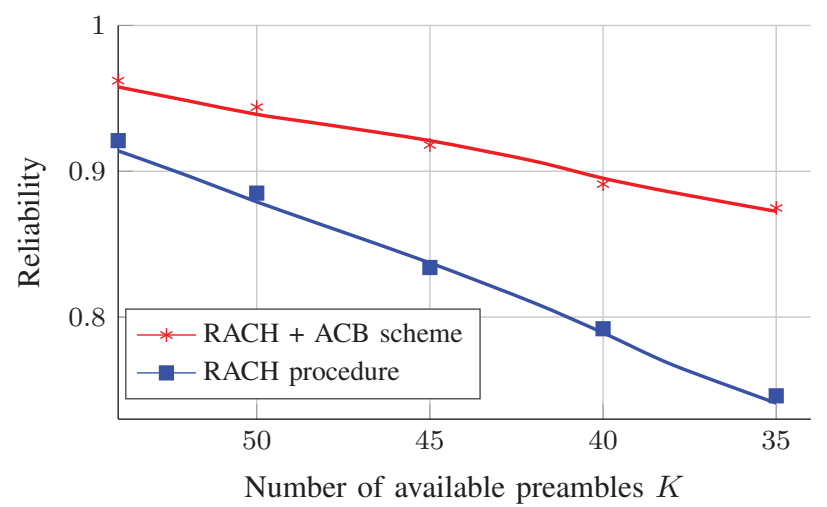

Fig. 8. Reliability achieved per IED as a function of the number of available preambles $K$ for contention-based access when $\mathrm{RACH}$ procedure is enhanced with the ACB scheme and when ACB is not applied.

occurrence of a burst and $\delta$ is related with the burst time duration. It can be seen that as the frequency of a burst increases, or equivalently $\gamma$ increases, the reliability decreases due to the higher attempt rate in the alarm state which leads to a surge of channel access attempts. In addition, as the length of each burst increases, or equivalently $\delta$ decreases, the reliability decreases since the IEDs remain longer in the alarm state.

Fig. 7 depicts the reliability achieved per IED as a function of the barring rate $r_{\text {th }}$ of the ACB scheme for a different number of IEDs. In particular, the reliability increases with a decreasing access barring rate; when the barring rate is set to a more restrictive value, it is more likely that new IED access requests are spread in time-subsequent attempts due to the barring backoff $B$. Although decreasing the barring rate may ease the congestion and improve access success probability, the average access delay for IED monitoring traffic is increased, especially for high number of access attempts. We aim to study and quantify the delay-reliability tradeoff for specific monitoring application requirements in future work.

Fig. 8 illustrates the reliability achieved per IED as a function of the number of available preambles $K$ for contentionbased access of the monitoring IEDs. It can be seen that reliability levels decrease as the number of available preambles decreases due to the lack of adequate access opportunities; however, a RACH procedure enhanced with the ACB scheme achieves higher reliability compared to the performance of the 
pure RACH procedure. This superior performance is important in use cases where the available preambles for contentionbased access are shared with the conventional LTE users in public cellular networks. An interesting tradeoff analysis would determine the optimal number of dedicated preambles for the competing traffic classes, given specific quality of service requirements.

\section{CONCLUSIONS}

In this paper, we propose an analytical framework for reliability analysis of massive monitoring traffic in cellularenabled distribution grids. Based on the Markov chain theory, we model the contention-based LTE random access operation enhanced with an ACB scheme for the connection establishment of a high number of IEDs. We further propose an MMPP traffic model to capture the IED transition from regular to alarm state. Based on the conducted analysis, we derive the reliability expression which depends on various $\mathrm{RACH}$ and ACB parameters and the monitoring IED traffic characteristics. Our analytical framework is validated through extensive simulations and the impact on the achieved reliability is evaluated under different network and traffic configurations. Since the contention-based random access procedure in recent 3GPP advancements tailored for the Internet of Things (IoT), i.e., LTE-M and NarrowBand IoT $^{2}$ (NB-IoT), follows its counterpart in LTE, the rationale of the proposed analytical model can also be applicable for the performance analysis of smart grid monitoring traffic using cellular IoT technologies.

Future investigations include the study of latency-reliability trade-offs based on specific monitoring application requirements and the quantification of the impact on background human-type traffic when present in a shared cellular system. We also aim to model the traffic dynamics of correlated transitions from regular to alarm state for different IEDs as a result of a widespread power grid cascading failure.

\section{ACKNOWLEDGMENT}

This work has been funded by projects ADVANTAGE under Grant 607774, P2P-SmarTest (646469), CellFive (TEC201460130-P) and the Catalan Government (2014-SGR-1551).

\section{REFERENCES}

[1] C. Kalalas, L. Thrybom, and J. Alonso-Zarate, "Cellular Communications for Smart Grid Neighborhood Area Networks: A Survey," IEEE Access, vol. 4, pp. 1469-1493, Apr. 2016.

[2] A. Laya, L. Alonso, and J. Alonso-Zarate, "Is the Random Access Channel of LTE and LTE-A Suitable for M2M Communications? A Survey of Alternatives," IEEE Commun. Surveys Tuts., vol. 16, pp. 416, Feb. 2014.

[3] 3GPP TR 37.868 v11.0.0, "Study on RAN improvements for MachineType Communications," Sep. 2011.

[4] 3GPP TS 36.331 V10.5.0, "Evolved Universal Terrestrial Radio Access (E-UTRA); Radio Resource Control (RRC)," Mar. 2012.

[5] J. P. Cheng, C. h. Lee, and T. M. Lin, "Prioritized Random Access with dynamic access barring for RAN overload in 3GPP LTE-A networks," in Proc. IEEE GLOBECOM Workshops (GC Wkshps), pp. 368-372, Dec. 2011.

${ }^{2}$ Due to the reduced bandwidth in NB-IoT, different preamble-design principles are adopted for the NB-IoT physical RACH compared to the legacy LTE physical RACH [16].
[6] R. G. Cheng, J. Chen, D. W. Chen, and C. H. Wei, "Modeling and Analysis of an Extended Access Barring Algorithm for Machine-Type Communications in LTE-A Networks," IEEE Trans. Wireless Commun., vol. 14, pp. 2956-2968, Jun. 2015.

[7] I. Leyva-Mayorga, L. Tello-Oquendo, V. Pla, J. Martinez-Bauset, and V. Casares-Giner, "Performance analysis of access class barring for handling massive M2M traffic in LTE-A networks," in Proc. IEEE Int. Conf. Commun. (ICC), pp. 1-6, May 2016.

[8] C. Karupongsiri, K. S. Munasinghe, and A. Jamalipour, "Random access issues for smart grid communication in LTE networks," in Proc. 8th Int. Conf. Signal Process. Commun. Syst. (ICSPCS), pp. 1-5, Dec. 2014.

[9] C.-H. Wei, R.-G. Cheng, and F. Al-Taee, "Dynamic radio resource allocation for group paging supporting smart meter communications," in Proc. IEEE 3rd Int. Conf. Smart Grid Commun. (SmartGridComm), pp. 659-663, Nov. 2012.

[10] G. Madueño, N. Pratas, Č. Stefanović, and P. Popovski, "Massive M2M access with reliability guarantees in LTE systems," in Proc. IEEE Int. Conf. Commun. (ICC), pp. 2997-3002, Jun. 2015.

[11] G. Madueño, J. Nielsen, D. Kim, N. Pratas, Č. Stefanović, and P. Popovski, "Assessment of LTE Wireless Access for Monitoring of Energy Distribution in the Smart Grid," IEEE J. Sel. Areas Commun., vol. 34, pp. 675-688, Mar. 2016.

[12] C. Kalalas, F. Vazquez-Gallego, and J. Alonso-Zarate, "Handling mission-critical communication in smart grid distribution automation services through LTE," in Proc. IEEE Int. Conf. Smart Grid Commun. (SmartGridComm), pp. 1-6, Nov. 2016.

[13] 3GPP, "Evolved Universal Terrestrial Radio Access (E-UTRA); Physical Channels and Modulation,' Mar. 2010.

[14] R. Nelson, Probability, Stochastic Processes, and Queueing Theory. Springer, New York, 1995.

[15] C. Kalalas, L. Gkatzikis, C. Fischione, P. Ljungberg, and J. AlonsoZarate, "Enabling IEC 61850 communication services over public LTE infrastructure," in Proc. IEEE Int. Conf. Commun. (ICC), pp. 1-6, May 2016.

[16] X. Lin, A. Adhikary, and Y. P. E. Wang, "Random Access Preamble Design and Detection for 3GPP Narrowband IoT Systems," IEEE Wireless Commun. Lett., vol. 5, pp. 640-643, Dec. 2016. 